\title{
Consumo midiático juvenil em experiências cosmopolitas: entre o entretenimento global e as práticas locais
}

\section{Youth media consumption within cosmopolitan experiences: between global entertainment and local practices}

\author{
Wilson Roberto Bekesas ${ }^{1}$ \\ Viviane Riegel ${ }^{2}$ \\ Renato Vercesi Mader ${ }^{3}$
}

Resumo Este artigo apresenta como proposta a discussão sobre a conexão entre consumo midiático juvenil e experiências cosmopolitas. Trata-se de parte constituinte do projeto de pesquisa Cosmopolitismos juvenis. Projeto Brasil. Etapa 1: Concepções e práticas cosmopolitas em jovens universitários brasileiros, sediado pela ESPM-SP, vinculado ao projeto internacional Cosmopolitismo Cultural dos Jovens. O recorte enfatizado está ancorado em uma base reflexiva sobre (1) o consumo midiático e a realidade do entretenimento global; e (2) as possibilidades de experiências cosmopolitas por meio das práticas locais. Analisando as narrativas de jovens universitários de São Paulo, verificamos a formação de experiências cosmopolitas prioritariamente estéticas, que poderiam ser denominadas de amadoras, como caminho para um processo reflexivo de alteridade.

Palavras-chave: Consumo midiático; Cosmopolitismo; Entretenimento; Global; Local

Escola Superior de Propaganda e Marketing - ESPM, São Paulo, SP, Brasil. E-mail: wbekesas@espm.br

2 Escola Superior de Propaganda e Marketing - ESPM, São Paulo, SP, Brasil.

E-mail: viviane_riegel@terra.com.br

${ }^{3}$ Escola Superior de Propaganda e Marketing - ESPM, São Paulo, SP, Brasil.

E-mail: rmader@espm.br 
Abstract This article presents as a proposal a discussion about the connection between youth media consumption and cosmopolitan experiences. This is a part of the research project "Youth cosmopolitanisms. Brazilian project. Step 1: Conceptions and cosmopolitan practices in Brazilian university students", hosted by ESPM-SP, linked to the international project "Youth cultural cosmopolitanism". The focus emphasized is anchored on a reflexive base on (1) media consumption and the reality of global entertainment; and (2) the possibilities of cosmopolitan experiences through local practices. Analyzing São Paulo's young people's narratives, we see the formation of primarily aesthetic cosmopolitan experiences, which could be called amateur, as a path to a reflexive process of alterity.

Keywords: Media consumption; Cosmopolitanism; Entertainment; Global; Local 


\section{Introdução}

Esse artigo tem como proposta apresentar a conexão entre consumo midiático juvenil e experiências cosmopolitas. Trata-se de parte constituinte do projeto de pesquisa Cosmopolitismos juvenis. Projeto Brasil. Etapa 1: Concepções e práticas cosmopolitas em jovens universitários brasileiros, sediado pela ESPM-SP, com apoio financeiro do CAEPM (Centro de Altos Estudos da ESPM).

Nossa proposta de discussão faz parte de um projeto internacional de pesquisa denominado Cosmopolitismo Cultural dos Jovens, que tem como objetivo desenvolver um estudo comparativo entre França, Canadá, Austrália e Brasil. É liderado pelo Departamento de Pesquisa de Previsão e Estatística do Ministério da Cultura e Comunicação da França e pelo Groupe d'Etude des Méthodes de l'Analyse Sociologique de la Sorbonne (GEMASS)/CNRS, Universidade de Paris 4.

O projeto inicial partiu da observação de dois fenômenos interdependentes: 1) a crescente internacionalização da produção e do consumo cultural, especialmente entre os jovens; e 2) a efervescência de produções e recepções artísticas locais, fenômeno que alguns sociólogos da cultura têm chamado de "indigenização" das práticas culturais (APPADURAI, 1994). A tensão entre esses dois movimentos resulta na discussão sobre a cultura global e seus diversos processos de localização.

A partir dessa perspectiva cultural da globalização, propomos a discussão sobre o consumo midiático dos jovens. A discussão surge por questões que se desenvolvem tanto a partir do entretenimento global, nos blockbusters e nos hits musicais presentes em diferentes partes do mundo, quanto da participação local, de indivíduos exercendo cidadania e se engajando em movimentos sociais, dentro do contexto do consumo midiático e da experiência cosmopolita. Um dos exemplos que poderíamos utilizar para ilustrar essa questão é a série de filmes estadunidense Hunger Games, lançada em 2012, sucesso de bilheteria ao redor do mundo. Da mesma forma que a série de livros e os produtos licenciados com os personagens da trama passaram a ser consumidos em larga escala mundial em diferentes formatos e meios, um dos símbolos 
da história, um gesto utilizado pela heroína protagonista como sinal de protesto contra o poder da capital, também foi utilizado por jovens em manifestações nas ruas da capital tailandesa contra o governo local no ano de 2014. Seria a utilização desse símbolo de entretenimento global como forma de participação local uma possibilidade de experiência cosmopolita?

Para responder tal questão, partimos da problematização do mercado do entretenimento e de suas dinâmicas, que são centrais na realidade contemporânea das culturas do consumo. Analisamos como o consumo midiático, inserido na lógica do entretenimento, forma mediações importantes na configuração de hábitos culturais que se movem entre as experiências globalizantes, unificadoras e hegemônicas, e seus processos de reapropriação em experiências locais. Nesse sentido, analisamos também como, a partir do consumo, é igualmente possível que ocorra participação local, em processos de construção de espaços públicos e exercícios de cidadania. Tal prática pode se relacionar aos fluxos globais, possibilitando aos indivíduos experiências do que se consideraria uma cidadania global. Assim, nossa discussão levanta a questão de como o consumo cultural deixa brechas para que os jovens construam representações de si e do outro inseridas em contextos "glocais" - de formação tanto global quanto local, sem determinação hierárquica entre os fluxos (ROBERTSON, 1992).

Nossa discussão está inserida no contexto contemporâneo, onde há aumento indiscriminado de tecnologias e modos de consumo midiático que pressupõem diferentes maneiras de ver, ouvir e falar com o mundo, entre plataformas e formatos com novos arranjos, que constituem as experiências cotidianas dos jovens sujeitos de nossa investigação. A quantidade de suportes tecnológicos, aliada a um sujeito histórico reconhecedor dos códigos midiáticos, deixa marcas de outras formas de disputas por significação que retroalimentam a centralidade da espetacularização da vida cotidiana, na acepção de Debord (1997). Analisando o conceito de espetáculo, Kellner (2003) o insere na cultura da mídia como forma cotidiana de interação social: 
Experience and everyday life are thus shaped and mediated by the spectacles of media culture and the consumer society. (...) Capitalist society separates workers from the products of their labor, art from life, and consumption from human needs and self-directing activity, as individuals inertly observe the spectacles of social life from within the privacy of their homes (KELLNER, 2003, p. 2-3).

Uma vez que as experiências cotidianas são moldadas e mediadas pelos espetáculos da mídia para estimular o consumo de experiências do mercado global contemporâneo, compreendemos que as linguagens de entretenimento são utilizadas, conforme a lógica da mass media entertainment industry (CASTRO, 2013).

Neste contexto, pretendemos discutir como os jovens constroem representações de si mesmos e de suas relações com o mundo através do consumo de bens culturais, sejam globalizados ou localizados. Procuramos evidências de que eles desenvolvem uma postura estética que pode ser considerada como processo de construção de um olhar para o outro. Em outras palavras, pretendemos entender o cosmopolitismo estético analisando o consumo cultural (música, TV, filmes, séries, jornais/ revistas, quadrinhos, livros, videogames, blogs/sites, redes sociais) e as experiências com a cultura global através dos quais os jovens constroem seus critérios de julgamento, conhecimento e imaginário do outro.

Para isso, desenvolvemos primeiramente uma discussão sobre o consumo midiático e a realidade do entretenimento global, seguida das reflexões sobre as possibilidades de experiências cosmopolitas em relação à dimensão estética. Analisamos, então, narrativas de jovens universitários de São Paulo como forma de compreender suas experiências cotidianas por seu consumo midiático, que remetem tanto a elementos de entretenimento quanto ao interesse por outras culturas e por contato com o Outro.

\section{Consumo midiático e entretenimento}

Autores como Lipovetsky e Serroy (2009) e Jacks e Escosteguy (2005) têm analisado as transformações do consumo midiático, correlacionando 
três perspectivas de novos agenciamentos comportamentais: o avanço das tecnologias de produção e o crescimento de novas plataformas midiáticas; o saber reflexivo do sujeito histórico; e o modo múltiplo e descentralizado de propagação de conteúdos.

Guardadas as devidas diferenças entre os pontos de vista dos respectivos autores, há uma confluência de pesquisas voltadas para o que Lipovetsky e Serroy (2009) nomearam como a era do "excesso de telas", problematizando aquilo que Jameson (1996) apontava como uma "virada cultural" a partir de 1960. Em outras palavras, o surgimento de novas configurações do capital vinculadas aos processos de estetização da vida cotidiana como "dominante cultural".

A discussão sobre o consumo midiático está relacionada aos estudos culturais, que transcendem a pesquisa dos meios, partindo para um contexto do circuito de produção, circulação e consumo da cultura midiática, sendo que a influência que os meios podem ter sobre as pessoas ou as culturas permanece relativa (JACKS; ESCOSTEGUY, 2005). A partir do desenvolvimento tecnológico, principalmente dos dispositivos digitais, os estudos sobre consumo midiático buscam a compreensão das relações dos sujeitos com os diferentes meios tecnológicos, utilizados simultaneamente por um processo de convergência midiática. Pelos meios digitais, os jovens contemporâneos produzem e consomem cultura e entretenimento como resultado tanto da familiaridade que possuem com as diferentes tecnologias quanto do acesso a diferentes dispositivos. Nesse caminho, a pesquisa Jovem e Consumo Cultural em Tempos de Convergência (JACKS, 2014) busca relacionar os elementos constitutivos das identidades juvenis em construção nos processos e práticas culturais através do consumo midiático.

A crescente disponibilidade tanto de modos de produção quanto de dispositivos midiáticos para consumo pressupõe diferentes apropriações de plataformas e formatos narrativos (gêneros), criando novos arranjos constitutivos das experiências cotidianas e juvenis no cenário contemporâneo. Junto a essa multiplicidade técnica está um sujeito histórico reconhecedor dos códigos, modos de produção, gêneros e narrativas. Ele 
deixa indícios de outras formas de disputas por significação que ocorrem principalmente pelo acesso à participação em redes imateriais que disponibilizam informação e entretenimento, num processo que Canclini (2009) denomina de desmaterialização da cultura pelas redes digitais.

A experiência desse sujeito pode igualmente remeter, por meio do hibridismo midiático, ao hibridismo presente no consumo cultural. No caso dos jovens, conforme aponta Canclini (2008), eles adquirem, por meio das telas, conhecimento e entretenimento combinados, sendo que, nesse processo de conectividade, há estímulo prioritário ao lazer e ao entretenimento.

Diante da centralidade do entretenimento através do consumo midiático, o processo publicitário interativo (COVALESKI, 2010) estabelece um foco na relação com o consumo cultural juvenil. Assim, é através do destaque alcançado no mercado global que se verificam as estratégias publicitárias de persuasão e entretenimento, inseridas nos produtos culturais em evidência. Interatividade, compartilhamento e acesso resultam em fluxos culturais globais que são consumidos e multiplicados em velocidade crescente. Fluxos que são apropriados tecnicamente pela indústria do entretenimento com a finalidade de atrair consumidores que buscam expandir as experiências de lazer para diversos meios. $\mathrm{O}$ entretenimento possibilita tanto a fruição estética como a distração intelectual, gerando eventualmente a formação de repertório cultural para esses jovens, assim como uma consequente e crescente demanda por opções de lazer. Exemplo desse fenômeno é a linha de produtos criada a partir dos livros de Harry Potter, que abrange desde os filmes, de grande impacto global, até os parques temáticos, as ruas e lojas de experiência dos cenários, os jogos virtuais, a interação com interfaces das histórias e muitos outros.

O entretenimento global e seus produtos midiáticos tornaram-se, dessa maneira, uma forma de cultura mundialmente popular (MIRRLEES, 2013) que resulta das forças transnacionais de consumo midiático, mas também de produção, distribuição, financiamento, codificação, decodificação e uso cultural que a rodeiam. Tais forças se concentram no que é denominado de mainstream. 
Martel (2012) mostra como a indústria do entretenimento norte-americana, voltada para o grande público, dominante e popular, forma a cultura mainstream. Em seu estudo, demonstra que são múltiplas as razões para esse domínio norte-americano, que estariam fundadas no fato de que o "sistema americano de produção de conteúdo é um modelo complexo, produto de uma história, de um território imenso e de uma imigração de todos os países, todas as línguas e todas as culturas" (MARTEL, 2012, p. 448). Tal cultura pode ter conotação positiva, no sentido de uma cultura que pode ser compartilhada por todos, ou negativa, no sentido de uma cultura hegemônica e dominante.

A aceitação das proposições feitas pela indústria de entretenimento global por diferentes realidades locais pode tanto sugerir que os produtos, mesmo parecendo exóticos, atraem o interesse dos públicos locais quanto mostrar um processo de consumo dos códigos culturais hegemônicos, com pouca adaptação ou resistência. Ao consumir produtos midiáticos de outras culturas, os indivíduos poderiam aumentar sua abertura a elas e cada vez aceitariam mais produtos estrangeiros. Alternativamente, os indivíduos também poderiam resistir a produtos culturais de outras culturas simplesmente por não poderem se relacionar com eles ou por preferirem valorizar seus códigos locais.

Nessa perspectiva, questionamos: seria essa a maneira de se estabelecer uma relação de consumo cultural de massa na lógica do mercado global, resultando em consumo cultural global padronizado? A interação ou a apropriação dos consumidores nesse processo, pela incorporação de seus comentários, conteúdos e significados, resultaria numa participação local e/ou individual nos fluxos globais do mercado de entretenimento? Tais questões nos remetem à experiência estética cotidiana dos sujeitos dentro de uma concepção cosmopolita da sociedade global.

\section{Concepção de cosmopolitismo vinculada à experiência estética}

O percurso dos estudos sobre cosmopolitismo na sociologia segue um intervalo que vai de Simmel (1971) a Beck (2006). Uma primeira 
hipótese sugere que esse percurso pode ser concebido pela passagem de uma sociologia do cosmopolitismo para um cosmopolitismo sociológico. Por sua vez, há outra hipótese que indica que, em virtude da pluralidade de ancoragens teóricas que marcam os estudos sobre o tema, o cosmopolitismo na sociologia remete a diversos percursos. A estratégia definida para mediações entre os estudos a ser considerada orienta-se pela análise imanente, tendo por elemento sistemático os aspectos internos de cada formulação.

Apresentamos aqui um conceito de cosmopolitismo vinculado ao de experiência estética para que possamos iniciar a discussão do consumo midiático em contextos culturais contemporâneos. Esse vínculo tem como base os trabalhos de Skrbis e Woodward (2013), que defendem a necessidade de se continuar a olhar para as manifestações e as possibilidades de cosmopolitismo nos encontros comuns na vida cotidiana das pessoas, e de Cicchelli e Octobre (2013), que afirmam que há necessidade de se empregar o conceito de cosmopolitismo em relação a situações comuns, como o consumo cultural cotidiano.

A natureza da relação entre cosmopolitismo e estética não estaria fundada em um conhecimento organizado, mas, inicialmente, em um acúmulo de momentos resultantes de encontros e experiências comuns, às vezes tanto efêmeras quanto parciais e organizadas como representações. A ligação com a alteridade seria, em primeira instância, estética (CICCHELLI; OCTOBRE, 2015): como no Facebook, a pessoa "curte" (ou não), comenta (ou não), compartilha (ou não) desde o último vídeo musical lançado por uma celebridade, como Beyoncé, até o impacto dos assassinatos ocorridos no Bataclan, em Paris, em novembro de 2015, marcando ambos os eventos nas linhas do tempo midiática e pessoal.

Essa relação do cosmopolitismo é muitas vezes reduzida às indústrias culturais/de entretenimento globais e a suas sugestões consumistas. Como no caso do turismo e lazer, onde a familiaridade com as culturas do mundo é vista como "superficial ou cosmética" (SASSATELLI, 2012, p. 235). A dimensão estética e cultural do cosmopolitismo seria acusada inclusive de manter o Outro como uma relação exótica, de 
reduzir a conexão com a alteridade ao consumo de bens e produtos em cenários urbanos padronizados (em situações de ansiedade de consumo pela efemeridade). Não se trata de buscar uma hierarquia entre as dimensões do cosmopolitismo, como se houvesse uma superior e outra inferior, ou uma mais autêntica e outra mais superficial. Há possibilidade de interseção de experiências e encontros nas diferentes dimensões do cosmopolitismo.

Viver em um mundo globalizado não implica necessariamente a adoção de uma perspectiva cosmopolita para o mundo ou de uma defesa da realização de suas aspirações (ética, política, cultural etc.). No entanto, a maioria das pessoas é capaz de desenvolver um cosmopolitismo estético, negando a ideia de uma única definição para o cosmopolitismo. Na literatura acadêmica, o cosmopolita é delineado como um sujeito altamente móvel, curioso, aberto e reflexivo, que se deleita com e deseja consumir a diferença, especialmente por ocasião de mobilidades internacionais, experiências em outros países ou outras formas de relações sociais transnacionais (HANNERZ, 1990; URRY, 1995). Nós empregamos o conceito de cosmopolitismo estético para analisar a inserção do sujeito no fenômeno da globalização, como um processo cultural transnacional que não apaga as culturas locais e que transmuta o sentimento de singularidade cultural nacional. Nessa perspectiva, o sujeito possui um sentimento estético que, graças à hibridização cultural de elementos de diversos horizontes, foi emancipado de um quadro anterior orientado localmente e de forma rígida.

A globalização mecânica do consumo cultural, ligada à crescente circulação de conteúdos culturais provenientes de diferentes continentes, deu origem a um cosmopolitismo estético definido, por um lado, por uma forte atração e curiosidade em relação a práticas culturais e produtos exóticos de diversos lugares, com referências localizadas, autênticas ou reinventadas, e, por outro lado, pela sua hibridação com formas culturais nacionais ou com apropriações individuais localizadas.

Isso conduz à necessidade de se analisar a morfologia individual e social deste esteticismo cosmopolita: individual em sua mobilização, ele 
é construído nas dobras singulares do social por experiências, emoções compartilhadas e imaginários, e pode ser definido como "uma disposição cultural envolvendo uma postura estética e intelectual de abertura para povos, lugares e experiências de diferentes culturas, especialmente aqueles de diferentes nações, ou, de um gosto para as margens mais amplas da experiência cultural" (CICCHELLI; OCTOBRE; RIEGEL, 2016, p. 57). Em um nível teórico, implica se concentrar em sentimentos, bem como conexões, como elemento central do imaginário pluralista e multicultural dentro e entre os grupos ou comunidades, uma vez que eles podem tanto tentar preservar suas culturas, costumes e identidades, quanto estar englobados em um mosaico de culturas hibridizadas. Implica, também, fazer a distinção entre o conhecimento objetivo de um indivíduo em relação a outros, com base em uma abertura de estruturas de recepção, representações e afetos.

Assim, a estética contemporânea torna-se cosmopolita: a) pelo reconhecimento, pelo indivíduo, da diferença entre os seus códigos estéticos vernaculares e aqueles dos produtos culturais estrangeiros consumidos; b) pela comparação dos códigos sem uma hierarquização necessária em uma escala de valor; c) pelo desenvolvimento de competências na manipulação desses diferentes códigos estéticos através de um processo de familiarização; e d) pelo desenvolvimento de uma intencionalidade no que diz respeito à descoberta da cultura da qual o produto emergiu.

Para o estudo de possibilidades de encontros cosmopolitas estéticos dos jovens, por meio de experiências, emoções e/ou imaginários vivenciados em seu cotidiano, desenvolvemos a análise do consumo cultural de jovens universitários em São Paulo, focando em seus aspectos midiáticos.

\section{Consumo midiático de jovens universitários em São Paulo}

No percurso metodológico definido para o projeto Cosmopolitismos juvenis. Projeto Brasil. Etapa 1: Concepções e práticas cosmopolitas em jovens universitários brasileiros, foram desenvolvidas duas etapas, 
a primeira qualitativa, realizada por meio de entrevistas individuais, e uma quantitativa, por meio de questionários. Para essa etapa, detalharemos as narrativas a partir das entrevistas. $\mathrm{O}$ roteiro definido para a entrevista semiestruturada explorou questões sobre consumo cultural e cultura global.

Os jovens selecionados para a etapa inicial do projeto de pesquisa são universitários, pelas possibilidades de formação e aproximação com questões globais desse público e pelo percurso delineado também para o início da pesquisa na França, fator que possibilita análises comparativas entre os dois contextos. O recorte feito nos dois países foi de jovens entre 18 e 24 anos. Em São Paulo, foram selecionados estudantes de instituições de ensino superior de três diferentes naturezas: pública, privada sem fins lucrativos e privada com fins lucrativos. Ao total, foram realizadas 40 entrevistas durante os meses de fevereiro e março de 2015.

Ao analisarmos as questões sobre consumo cultural, destacam-se principalmente o consumo de música, redes sociais, e sites/blogs. A relação do tempo, no entanto, denota alta prioridade para o uso de redes sociais, que são utilizadas praticamente em tempo contínuo e se sobressaem em relação a outros meios para consumo cultural em geral. Esta concentração mostra a prioridade de consumo de mídias digitais, bem como as relações de socialização dos jovens através das redes sociais. Notamos nos diálogos com os jovens que o intervalo de tempo entre acordar e conectar-se às redes sociais é curto e representa a primeira e primordial forma de contato com informações e entretenimento para eles. Da mesma forma que o consumo cultural concentra-se em produtos digitais, verificamos que os hardwares mais utilizados para produtos culturais distintos (música, literatura, filmes etc.) pelos jovens estudantes de São Paulo são computadores pessoais e smartphones. Há também o consumo de televisão, mas, principalmente, a partir de conexões privadas, incluindo acesso à internet.

O consumo de diferentes produtos culturais é principalmente de origem nacional, mas também ocorre o consumo de origem estrangeira, principalmente de produtos dos Estados Unidos. Essa relação com 
a cultura norte-americana pode ser explicada tanto pelo domínio da cultura global por produtos desse país - na perspectiva mainstream da indústria cultural - quanto pelo conhecimento desses jovens do idioma inglês. A partir do consumo de mídia, principalmente norte-americana, buscamos a possibilidade de reflexividade dos jovens com outras culturas, o que poderia desenvolver neles uma postura cosmopolita.

Para estudar essa postura cosmopolita, analisamos as narrativas desses jovens de São Paulo em relação à dimensão estética do consumo midiático. Sobre o debate a respeito da aplicação do cosmopolitismo estético, destacamos que não há garantia de que a expansão dos horizontes culturais, o desenvolvimento de competências e habilidades culturais e habilidades hermenêuticas se traduzam necessariamente no surgimento de um senso de responsabilidade para com o mundo. No entanto, a introdução da dimensão estética marca uma grande mudança na forma como se pode considerar o cosmopolitismo, como um estilo de vida relacionado ao consumo. A questão da cidadania global não gira apenas em torno da participação política e engajamento cívico, mas também em torno dos gostos cosmopolitas, estilos e padrões de consumo (GERMANN MOLZ, 2011). Assim como afirma Papastergiadis (2012), o cosmopolitismo estético-cultural é fundamental, pois se baseia na capacidade individual e coletiva de construção de uma imagem do mundo, com a arte sendo menos uma forma de representar a verdade do mundo e mais uma ferramenta para imaginar sua realidade.

As configurações personificadas pelos jovens em suas experiências cotidianas mostram graus variados de reflexividade. O cosmopolitismo a partir do consumo pode ser desconectado da reflexividade e da abertura à alteridade consciente. Em outros casos, esta reflexividade é muito mais presente e comprometida com uma introspecção crítica para si e para a cultura sob a forma de comparação explícita, que pode alimentar a produção de estereótipos e de ferramentas de comparação.

Podemos ilustrar a produção de estereótipos na narrativa de Angélica, 20 anos, que afirma que seu consumo de filmes e informações é globalizado, prioritariamente em inglês e de origem norte-americana. 
Para a estudante, ser conectada ao mundo é ter conexão com os principais meios de comunicação globais e da cultura mainstream. De forma semelhante, Oliver, de 21 anos, afirma que somente consome produtos culturais norte-americanos, que fazem parte da cultura mainstream, e afirma que o faz por diversão e por se sentir parte do mundo ao compartilhar os mesmos gostos e práticas que milhões de pessoas também experienciam ao redor do mundo.

Já a produção de ferramentas de comparação pode ser encontrada na narrativa de Fabio, 21 anos, para quem o consumo de música e televisão se resume a conteúdo de outros países, principalmente Estados Unidos e Inglaterra. Ele entende que deveria se sentir mais conectado a outras culturas e o faz principalmente pelo consumo midiático, buscando nessa interação possibilidades de aprendizado e de diversão.

Em contraponto ao consumo mainstream e na busca por produzir comparações, temos o exemplo na narrativa de Bibiana, 22 anos, que demonstra uma perspectiva crítica aos produtos culturais mainstream ao declarar que não consome a variedade norte-americana de grandes sucessos cinematográficos, embora frequente as salas de cinema regularmente, em média três vezes por semana. Para ela, cinema representa mundo, e mundo abraça culturas, povos e idiomas distantes e estranhos à cultura brasileira. Cita o cinema europeu contemporâneo, o cinema indiano, asiático e oriental como exemplos que a atraem.

Nessas formas de comparação dos produtos culturais com emoções, sentimentos identificados e conhecimento que permanece é que o consumo misturado de produtos nacionais e estrangeiros resulta na figura do cosmopolita amador (CICCHELLI; OCTOBRE; RIEGEL, 2016). Essa figura é diferente de outros amadorismos porque trata de compromissos transitórios e reversíveis nos repertórios culturais que não são estritamente definidos pela educação, como sugerido pelo modelo de Bourdieu (2007). Além disso, este amador está intimamente ligado à crescente importância da socialização geracional horizontal entre pares no contexto digital, de forma que este amadorismo serve também como um recurso para a autoconstrução desses jovens. 
Percebemos a figura do cosmopolita amador na narrativa de Rafael, 22 anos, ao situar que cinema e televisão estão completamente fora de seu arco de consumo cultural, focando seu gosto e preferência em música e videogames como canais próprios de cultura e entretenimento, onde ele tem a possibilidade de trocar informações, conhecer referências de outros locais e encontrar pares de outros países e culturas. Rafael situa o consumo ocasional, não sistemático, de séries a partir de amostras exibidas na internet e que o agradam. Revela-se um ávido consumidor de produtos culturais para seu entretenimento que tenham a possibilidade de troca e construção, daí a predileção pelo consumo digital, que lhe dá liberdade de interação.

Assim, neste amadorismo, a questão não é de medição do grau de conhecimento atual sobre as culturas de outros, mas o grau percebido de proximidade - ou distância - com a alteridade em um contexto de fluxo massivo de bens culturais. Como no caso de Bibiana, que considera que o cinema americano não é interessante, ao passo que obras de outros países, justamente por apresentarem uma perspectiva cultural diferente, por mais ficcional que seja a obra, potencializam sobremaneira seu interesse e consumo. No caso de Rafael, ele demonstra profundo conhecimento do que ocorre tanto no cenário da música contemporânea americana quanto no do videogame e considera ambas as formas importantes para consumo de entretenimento e contato com a cultura americana.

Por fim, no caso de Issaka, 24 anos, que nasceu no Níger e vive no Brasil há oito anos, a curiosidade é um fator determinante. Passou por diferentes países e fala diversos idiomas. Sua mobilidade e suas experiências lhe permitem a construção de mapas, pelo cuidado com os lugares que pretende visitar e compartilhar da vida local, temporariamente. Como ele mesmo se define, é um viajante, mas quer se inscrever nos locais para vivê-los: "eu gosto de conhecer um pouco da história [desses lugares], de planejar um pouco, conhecer [para] não estranhar”. Nas afinidades com outros jovens que vivem nos lugares visitados e vividos, ele constrói uma relação de emoções compartilhadas. 
Surge, então, nossa reflexão a partir da narrativa desses jovens: a disposição para troca com o outro além da presença, com intensa utilização dos meios digitais. O quadro que se apresenta é de jovens, sujeitos em conexão, que fazem da rede um ambiente de possibilidades sobre o que querem experimentar. Como cartógrafos, usam suas redes sociais e outros ambientes para construir outro tipo de mapa, o das afinidades que permitem o reconhecimento dos outros. Suas descobertas parecem buscar confirmação do que investigaram pela rede; in loco, navegadores que são, procuram contato físico/local para a construção de suas narrativas investigatórias. O que procuram? Talvez mais sobre si mesmos, talvez mais sobre o mundo que os envolve, usando o outro como mais um espelho diante de um mundo de diversas aparências e reflexos.

\section{Considerações finais}

As relações de consumo cultural midiático estudadas seguem a lógica do mercado global, principalmente nos meios digitais, centrados nas instituições e marcas norte-americanas. $\mathrm{O}$ consumo nestes meios não é, no entanto, padronizado, uma vez que há possibilidades de incorporação de comentários, conteúdo e significado a partir dos signos locais, o que nos leva a observar que há tanto padrões massivos para o consumo quanto alternativos e únicos, como pudemos observar nos perfis aqui relatados a partir das entrevistas realizadas. De forma que, enquanto alguns jovens pesquisados consomem o padrão norte-americano de produtos culturais hegemônicos, há também outros que se recusam a consumir este modelo.

Esta recusa clara ao produto cultural norte-americano não foi percebida com a mesma intensidade em relação à produção de qualquer outro país. A produção nacional de países menos expressivos em volume de produtos culturais não é tão conhecida a ponto de estabelecer um padrão de recusa. Assim, essa relação se concentra em aspectos da cultura norte-americana e seus produtos midiáticos mainstream. A recusa à produção norte-americana em dado gênero de produção cultural, como o cinema, por exemplo, não significa, no entanto, distância das relações 
com produtos originários desse país, principalmente se avaliamos o consumo cultural digital e suas principais mídias.

Cinema, música, revistas em quadrinhos, livros, programas de televisão e outros produtos culturais são formas de contato com a cultura global a partir de suas realidades locais de produção. Padrões, especialmente no que diz respeito a consumo cultural e, mais especificamente, o consumo que ocorre a partir de suportes midiáticos, são especificamente direcionadores, pois é na padronização de audiência e conteúdos que a indústria cultural concentra sua produção de bens de consumo cultural. Diante do padrão global, há, no entanto, formas diferentes de consumo local, o que pode desenvolver diferentes posturas reflexivas e formas de participação.

A discussão proposta do consumo midiático pelos jovens universitários da cidade de São Paulo (uma cidade global, quiçá cosmopolita) a partir de uma perspectiva cultural nos revela que há tanto presença quanto uso dos produtos globais de entretenimento, mas que não necessariamente essa experiência estética se transforma em reflexividade local. Assim, consideramos que esses jovens seriam o que denominamos de cosmopolitas amadores. É importante destacar que esses jovens possuem um perfil específico em relação ao quadro geral da população da cidade de São Paulo e do Brasil, pois representam ainda uma minoria socioeconômica que realiza formação universitária. Sendo assim, o projeto de pesquisa busca ampliar, em suas outras etapas, a investigação para outros perfis de jovens, assim como a comparação com outras cidades do país.

O consumo midiático de produtos culturais, notadamente os globais, mainstream, poderia ser uma maneira de desenvolvimento de reflexividades múltiplas nos jovens. Além disso, interesses e afinidades por outras culturas, por suas diferenças e desafios marcam também uma intenção de olhar para o Outro. Pela reflexividade, seria possível construir uma postura cosmopolita, uma perspectiva com interpretações estrangeiras, um passo em relação à alteridade. Essas são as questões que seguem em nossa investigação das experiências de consumo cultural dos jovens brasileiros. 


\section{Referencias bibliográficas}

APPADURAI, A. Disjunção e diferença na economia global. In: FEATHERSTONE, M. (Org.). Cultura global. Petrópolis: Vozes, 1994.

BECK, U. Qu'est-ce que le Cosmopolitisme? Paris: Aubier, 2006.

BOURDIEU, P. A Distinção: Crítica Social do Julgamento. São Paulo: Edusp, 2007.

CANCLINI, N. G. Consumo, Acesso e Sociabilidade. Comunicação, Mídia e Consumo, São Paulo, v. 6, n. 16, p. 111-127, jul. 2009.

Leitores, Espectadores e Internautas. São Paulo: Iluminuras, 2008.

CASTRO, G. Entretenimento, sociabilidade e consumo nas redes sociais: cativando o consumidor-fã. In: ROCHA, R.; CASAQUI, V. (Org.). Estéticas midiáticas e narrativas do consumo. Porto Alegre: Sulina, 2013.

CICCHELli, V.; OCTOBRE, S. A Cosmopolitan Perspective of Globalization: cultural and aesthetic consumption among young people. Studies of Changing Societies: comparative and interdisciplinary focus, SCS Journal. v. 3 (7), p. 3-23, 2013.

Sur le Cosmopolitisme Esthétique chez les Jeunes. Le Débat. n. 183, p. 101109, Paris: Gallimard, 2015.

CICCHELLI, V.; OCTOBRE, S.; RIEGEL, V. Reflections on global culture and aesthetic cosmopolitanism. Global Studies Journal, v. 9, n. 1, p. 55-70, 2016.

COVALESKI, R. Publicidade Híbrida. Curitiba: Maxi, 2010.

DEBORD, G. A Sociedade do Espetáculo: comentários sobre a sociedade do espetáculo. Rio de Janeiro: Contraponto, 1997.

GERMANN MOLZ, J. Cosmopolitanism and Consumption. In: ROVISCO, M.; NOWICKA, M. (Eds.). The Ashgate Research Companion to Cosmopolitanism. [s.l.]: Routledge, 2011. p. 33-52.

HANNERZ, U. Cosmopolitans and locals in world culture. In: FEATHERSTONE, M. (Ed.). Global Culture. Londres: Sage, 1990.

JACKS, N. et al. Jovem e Consumo Midiático: dados preliminares do estudo piloto e da pesquisa exploratória. In: XXIII ENCONTRO ANUAL DA COMPÓS. Anais... Belém, 2014.

; ESCOSTEGUY, A. C. Comunicação e Recepção. São Paulo: Hacker, 2005.

JAMESON, F. Pós-modernismo: a lógica cultural do capitalismo tardio. São Paulo: Ática, 1996.

KELLNER, D. Media Spectacle. Califórnia: Routledge, 2003.

LIPOVETSKY, G.; SERROY, J. A Tela Global: mídias culturais e cinema na era hipermoderna. Porto Alegre: Sulina, 2009.

MARTEL, F. Mainstream: a guerra global das mídias e das culturas. São Paulo: Civilização Brasileira, 2012.

MIRRLEES, T. Global Entertainment Media: between cultural imperialism and cultural globalization. Nova York: Routledge, 2013.

PAPASTERGIADIS, N. Cosmopolitanism and Culture. Cambridge: Polity, 2012.

ROBERTSON, R. Globalization: social theory and global culture. Londres: Sage, 1992. 
SASSATELLI, M. Festivals, Museums, Exhibitions: aesthetic cosmopolitanism in the cultural public sphere. In: DELANTY, Gerard (Ed.). Handbook of Cosmopolitan Studies. Londres: Routledge, 2012. p. 233-244.

SIMMEL, G. On Individuality and Social Forms. Chicago: University of Chicago Press, 1971.

SKRBIS, Z.; WOODWARD, I. Cosmopolitanism: uses of the idea. Londres: Sage, 2013. URRY, J. Consuming Places. Londres: Routledge, 1995.

\section{Sobre os autores}

Wilson Roberto Bekesas - Doutor em Comunicação e Semiótica pela Pontifícia Universidade Católica de São Paulo (PUC-SP). Professor no curso de graduação em Publicidade e Propaganda na Escola Superior de Propaganda e Marketing (ESPM-SP).

Viviane Riegel - Doutoranda no Programa de Pós-graduação em Comunicação e Práticas de Consumo da Escola Superior de Propaganda e Marketing (ESPM-SP). Bolsista Capes/Prosup.

Renato Vercesi Mader - Doutorando no Programa de Pós-graduação em Comunicação e Práticas de Consumo Escola Superior de Propaganda e Marketing (ESPM-SP).

Data de submissão: 31/08/2015

Data de aceite: 04/03/2016 